\title{
Comparison of Okumura, Hata and COST-231 Models on the Basis of Path Loss and Signal Strength
}

\author{
Yuvraj Singh
}

\begin{abstract}
Radio propagation is essential for emerging technologies with appropriate design, deployment and management strategies for any wireless network. It is heavily site specific and can vary significantly depending on terrain, frequency of operation, velocity of mobile terminal, interface sources and other dynamic factor. Accurate characterization of radio channel through key parameters and a mathematical model is important for predicting signal coverage, achievable data rates, BER and Antenna gain.

Large scale path loss modeling plays a fundamental role in designing both fixed and mobile radio systems. Predicting the radio coverage area of a system is not done in a standard manner. Wireless systems are expensive systems. Therefore, before setting up a system one has to choose a proper method depending on the channel's BTS antenna height gain. By proper selecting the above parameters there is a need to select the particular communication model which show good result by considering these parameters.
\end{abstract}

\section{INTRODUCTION}

Wireless access network has becoming vital tools in maintaining communications especially at home and workplaces due to communication models. Propagation models can be classified mainly into two extremes, i.e. fully empirical models and Deterministic models. There are some models which have the characteristics of both types. Those are known as Semi-empirical models. Empirical models are based on practically measured data. Since few parameters are used, these models are simple but not very accurate. The models which are categorized as empirical models for macro cellular environment. These include Hata model, Okumura model, COST-231 Hata model. On the other hand, deterministic models are very accurate. Some of the examples include Ray Tracing and Ikegami model. As mentioned earlier, semiempirical models are based on both empirical data and deterministic aspects. Cost-231 Walfisch-Ikegami model is categorized as a semi empirical model. All these models estimate the mean path loss based on parameters such as antenna heights of the transmitter and Receiver, distance between them, etc. These models have been extensively validated for mobile networks. Most of these models are based on a systematic interpretation of measurement data obtained in the service area [1][2][3][4][5][6].

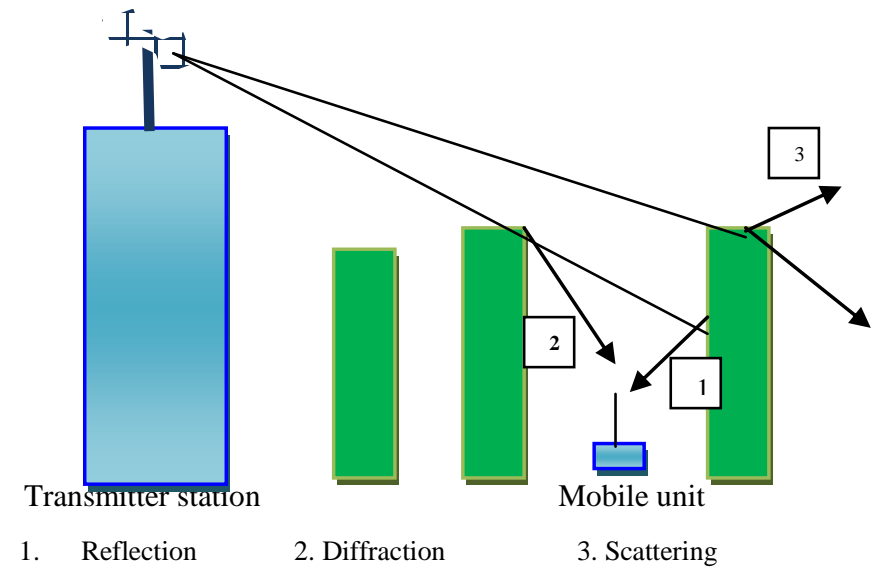

Fig Mechanism of propagation

\section{OKUMURA MODEL}

This is the most popular model that being used widely The Okumura model for Urban Areas is a Radio propagation model that was built using the data collected in the city of Tokyo, Japan. The model is ideal for using in cities with many urban structures but not many tall blocking structures. The model served as a base for Hata models. Okumura model was built into three modes which are urban, suburban and open areas. The model for urban areas was built first and used as the base for others. Clutter and terrain categories for open areas are there are no tall trees or buildings in path, plot of land cleared for $200-400 \mathrm{~m}$. For examples at farmland, rice fields and open fields. For suburban area the categories is village or highway scattered with trees and houses, few obstacles near the mobile. Urban area categories is built up city or large town with large buildings and houses with two or more storey or larger villager with close houses and tall, thickly grown trees.

Formula for Okumura Model is expressed below:

$L_{m}(d B)=L_{F}(d)+A_{m u}(f, d)-G\left(h_{r}\right)-G\left(h_{t}\right)-G_{A R E A}$

Where;

$\mathrm{L}_{\mathrm{m}} \quad=$ (i.e., median) of path loss

$\mathrm{L}_{\mathrm{F}}(\mathrm{d}) \quad=$ free space propagation path loss.

$\mathrm{A}_{\mathrm{mu}}(\mathrm{f}, \mathrm{d})=$ median attenuation relative to free space

$\mathrm{G}\left(\mathrm{h}_{\mathrm{b}}\right) \quad$ = base station antenna height gain factor

$\mathrm{G}\left(\mathrm{h}_{\mathrm{m}}\right)=$ mobile antenna height gain factor

$\mathrm{G}\left(\mathrm{h}_{\mathrm{b}}\right) \quad=20 \log \left(\mathrm{h}_{\mathrm{b}} / 200\right) 1000 \mathrm{~m}>\mathrm{h}_{\mathrm{b}}>30 \mathrm{~m}$

$\mathrm{G}\left(\mathrm{h}_{\mathrm{m}}\right) \quad=10 \log \left(\mathrm{h}_{\mathrm{m}} / 3\right) \mathrm{h}_{\mathrm{m}}<=3 \mathrm{~m}$

$\mathrm{G}\left(\mathrm{h}_{\mathrm{m}}\right) \quad=20 \log \left(\mathrm{h}_{\mathrm{m}} / 3\right) 10 \mathrm{~m}>\mathrm{h}_{\mathrm{m}}>3 \mathrm{~m}$ 
GAREA: gain due to type of environment given in suburban, urban or open areas Correction factors like terrain related parameters can be added using a graphical form to allow for street orientation as well as transmission in suburban and open areas and over irregular terrain. Irregular terrain is divided into rolling hilly terrain, isolated mountain, general sloping terrain and mixed land-sea path. The terrain related parameters that must be evaluated to determine the various corrections factors [6][7][8][9][10].

\section{HATA MODEL}

Hata established empirical mathematical relationships to describe the graphical information given by Okumura. Hata's formulation is limited to certain ranges of input parameters and is applicable only over quasi-smooth terrain. The mathematical expression and their ranges of applicability are as follows [3][5][9]

\section{Carrier Frequency: $150 \mathrm{MHz} \leq \mathrm{f}_{\mathrm{c}} \leq 1500 \mathrm{MHz}$}

Base Station (BS) Antenna Height: $30 \mathrm{~m} \leq \mathrm{h}_{\mathrm{b}} \leq 200 \mathrm{~m}$

Mobile Station (MS) Antenna Height: $1 \mathrm{~m} \leq \mathrm{h}_{\mathrm{m}} \leq 10 \mathrm{~m}$

Transmission Distance: $1 \mathrm{~km} \leq \mathrm{d} \leq 20 \mathrm{~km}$

$\mathrm{A}+\mathrm{B} \log _{10}(\mathrm{~d})$

for urban areas

$\mathrm{L}_{\mathrm{p}}(\mathrm{dB})=\mathrm{A}+\mathrm{B} \log _{10}(\mathrm{~d})-\mathrm{C}$ for suburban area

$A+B \log _{10}(d)-D \quad$ for open area

Where:

$A=69.55+26.161 \log _{10}\left(f_{c}\right)-13.82 \log _{10}\left(h_{b}\right)-a\left(h_{m}\right)$

$\mathrm{B}=44.9-6.55 \log _{10}\left(\mathrm{~h}_{\mathrm{b}}\right)$

$\mathrm{C}=5.4+2\left[\log _{10}\left(\mathrm{f}_{\mathrm{c}} / 28\right)\right]^{2}$

$\mathrm{D}=40.94+4.78\left[\log _{10}\left(\mathrm{f}_{\mathrm{c}}\right)\right]^{2}-18.33 \log _{10}\left(\mathrm{f}_{\mathrm{c}}\right)$

Where, $\mathrm{a}\left(\mathrm{h}_{\mathrm{m}}\right)=$

$\left[1.1 \log _{10}\left(f_{c}\right)-0.7\right] h_{m}-\left[1.56 \log _{10}\left(f_{c}\right)-0.8\right]$ for medium or small cities

$8.29\left[\log _{10}\left(1.54 \mathrm{~h}_{\mathrm{m}}\right)\right]^{2}-1.1$ for large city and $\mathrm{f}_{\mathrm{c}} \leq 200 \mathrm{MHz}$

$3.2\left[\log _{10}\left(11.75 h_{m}\right)\right]^{2}-4.97$ for large city and $\mathrm{f}_{\mathrm{c}} \geq 400 \mathrm{MHz}$

\section{COST-231 MODEL}

Most future PCS systems are expected to operate in the 1800$2000 \mathrm{MHz}$ frequency band. It has been shown that path loss can be more dramatic at these frequencies than those in the $900 \mathrm{MHz}$ range. Some studies have suggested that the path loss experienced at $1845 \mathrm{MHz}$ is approximately $10 \mathrm{~dB}$ larger than those experienced at $955 \mathrm{MHz}$, all other parameters being kept constant. The COST231-Hata model extends Hata's model for use in the $1500-2000 \mathrm{MHz}$ frequency range, where it is known to underestimate path loss. The model is expressed in terms of the following parameters [9][10][13][17]

Carrier Frequency $\left(f_{c}\right)_{1500}-2000 \mathrm{MHz}$

BS Antenna Height (hb) 30-200 m

MS Antenna Height $\left(\mathrm{h}_{\mathrm{m})} 1-10 \mathrm{~m}\right.$

Transmission Distance (d) 1-20 km
The path loss according to the COST-231-Hata model is expressed as:

$\mathrm{L}_{\mathrm{p}}(\mathrm{dB})=\mathrm{A}+\mathrm{B} \log 10(\mathrm{~d})+\mathrm{C}$

Where;

$$
\begin{aligned}
A & =46.3+33.9 \log 10\left(f_{c}\right)-13.28 \log 10\left(h_{b}\right)-a\left(h_{m}\right) \\
B & =44.9-6.55 \log 10\left(h_{b}\right) \\
C & =0 \quad \text { for medium city and suburban areas } \\
& 3 \quad \text { for metropolitan areas }
\end{aligned}
$$

\section{Calculation of Path loss}

The common representation formula of different communication models is [16][17][18]

PL $(d)=$ PL $(\mathrm{d} 0)+10 \mathrm{n} \log 10\left(\mathrm{~d} / \mathrm{d}_{0}\right)$ where

$\mathrm{d}=$ Distance between Transmitter station and Mobile station

$\mathrm{d}_{\mathrm{o}}=$ Reference point

$\mathrm{n}=$ Path loss exponent

\section{Calculation of signal strength}

The Received Signal Strength Indicator (RSSI) or Signal Strength is a measure of how strong the most recent signal was when it reached its destination. The RSSI value ranges from 0 to 255 . Higher RSSI values indicate a stronger signal. Reliable communication can best be achieved with RSSI values greater than 70 . If the RSSI is too low the wireless communications may become intermittent or fail entirely. The received signal strength for Okumura model, Hat model and COST-231 model can be calculated as

$P_{r}=P_{t}+G_{t}+G_{r}-P L-A$

Where

$P_{r}$ is received signal strength in $d_{m}$.

$P_{t}$ is transmitted power in $d_{m}$.

$\mathrm{G}_{\mathrm{t}}$ is transmitted antenna gain in $\mathrm{dB}_{\mathrm{m}}$.

$G_{r}$ is received antenna gain in $d_{B}$.

PL is total path loss in $\mathrm{dB}_{\mathrm{m}}$.

$A$ is connector and cable loss in $\mathrm{dB}_{\mathrm{m}}$.

In this work, connector and cable loss are not taken into consideration [5][18][20][21][22]

\section{RESULTS}

\subsection{Comparison of Okumura, Hata and Cost-231 models based on Path Loss}

Since attenuation is also the main cause of path loss which can be described as:

$A=16.5+15 \log (f / 100)-0.12 d$

Where; $\mathrm{f}=$ frequency of operation, $\mathrm{d}=$ distance travelled. 


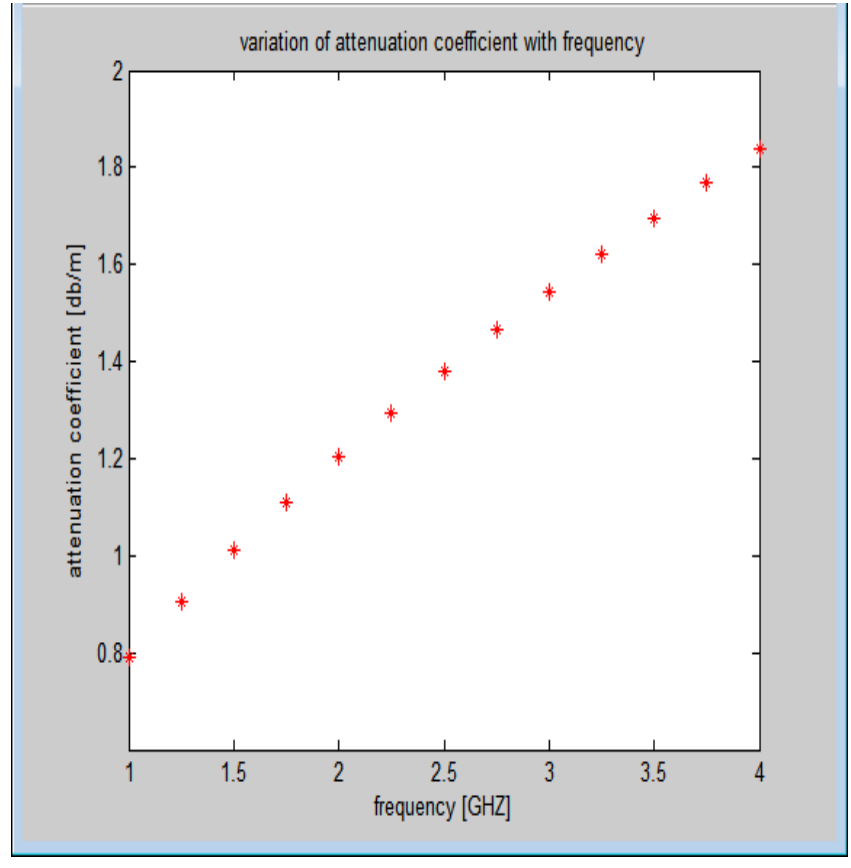

Fig1.Variation of attenuation coefficient with frequency.

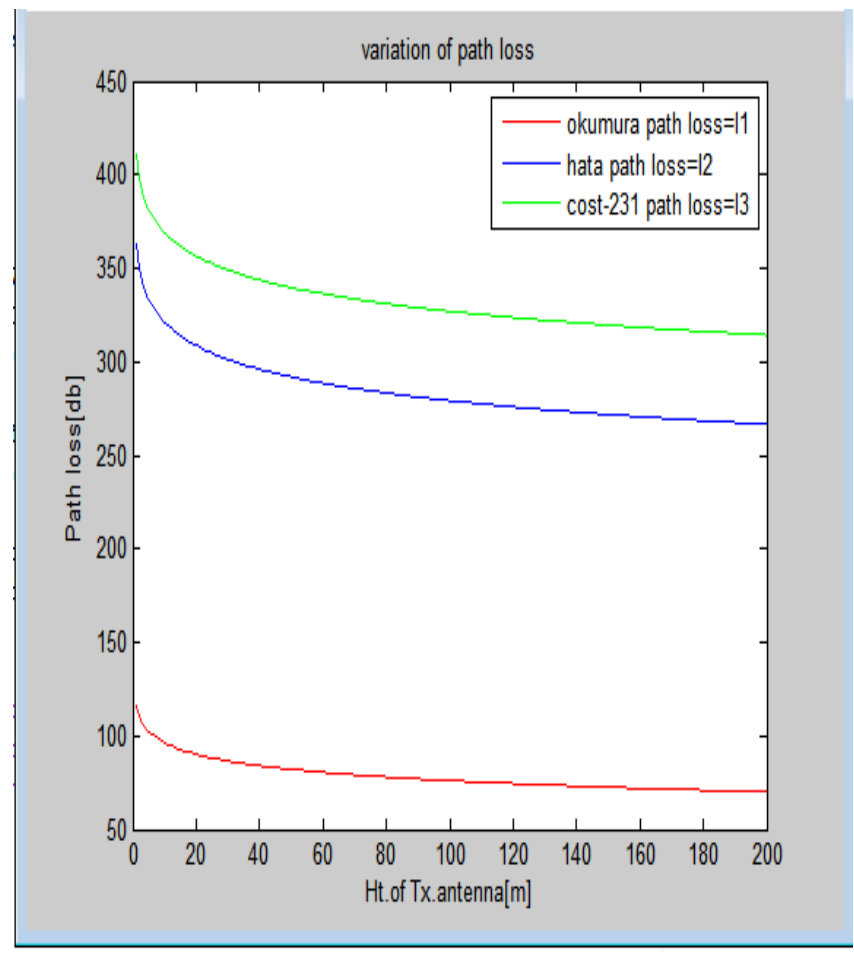

Fig2.Comparison of path loss of communication models with respect to height of transmitter antenna.

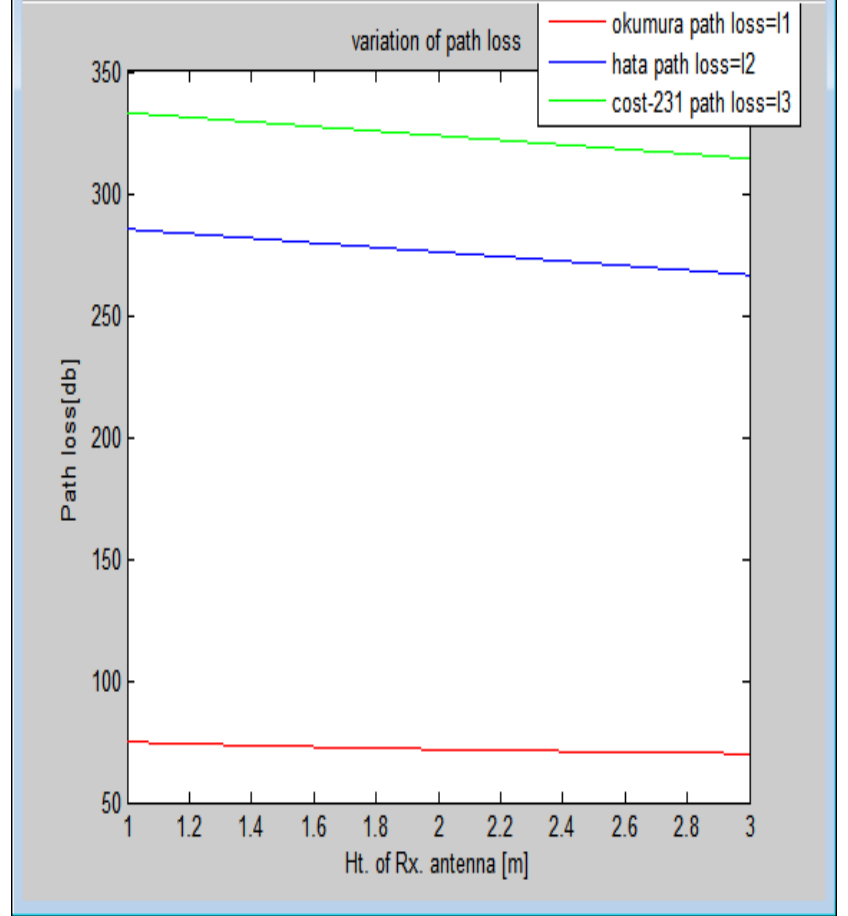

Fig3.Comparison of path loss of communication models with respect to height of receiver antenna.

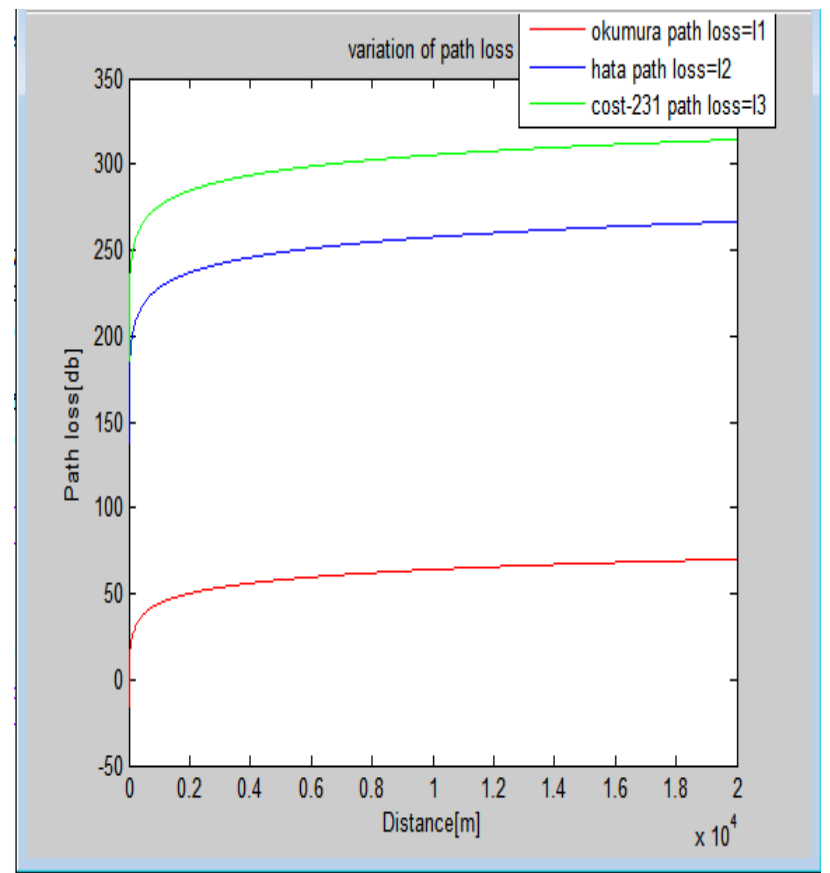

Fig4. Comparison of path loss of communication models with respect to transmission distance.

Table1.Comparison of path loss of communication models with respect to distance

\begin{tabular}{|l|l|l|l|}
\hline Distance(km) & $\begin{array}{l}\text { Okumura } \\
\text { path } \\
\text { loss(db) }\end{array}$ & $\begin{array}{l}\text { Hata path } \\
\text { loss(db) }\end{array}$ & $\begin{array}{l}\text { Cost-231 } \\
\text { path } \\
\text { loss(db) }\end{array}$ \\
\hline 1 & 44.03 & 227.74 & 275.51 \\
\hline 10 & 64.03 & 257.57 & 305.34 \\
\hline 20 & 70.05 & 266.54 & 314.32 \\
\hline
\end{tabular}




\subsection{Comparison of Okumura, Hata and Cost-231 models based on signal strength}

Since the comparison of communication models based on signal strength which considered the correction factor into account is

$\mathrm{C}=(1.1 \log -0.7) \mathrm{h}_{\mathrm{m}}-(1.56 \log \mathrm{f}-0.8)$

Where; $\mathrm{h}_{\mathrm{m}}=$ height of receiver antenna.

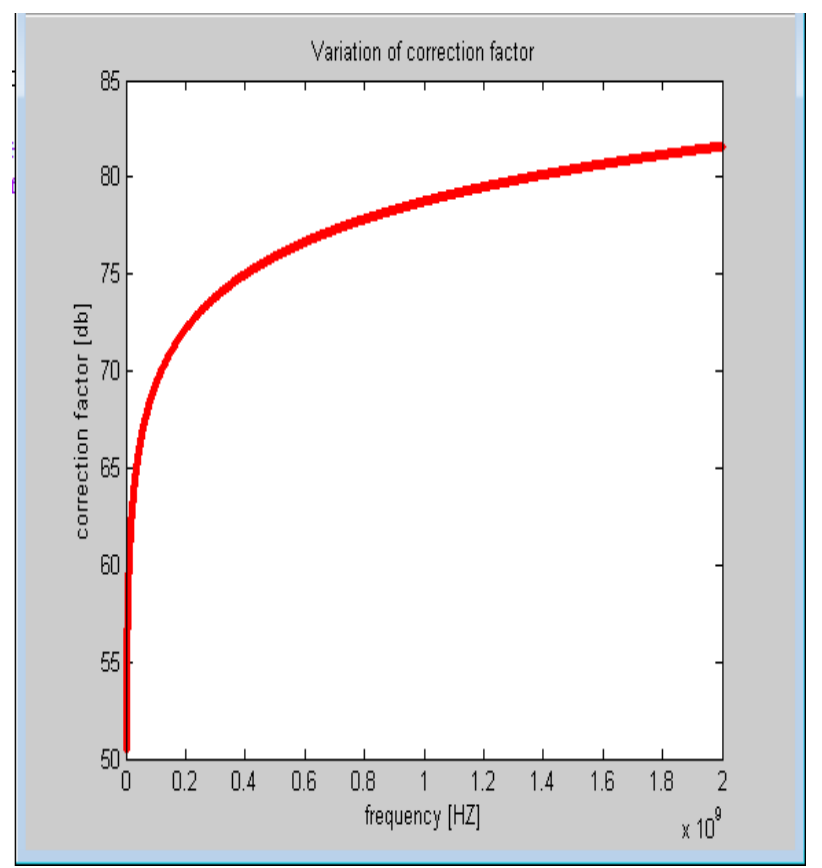

Fig 5.Variation of correction factor with frequency.

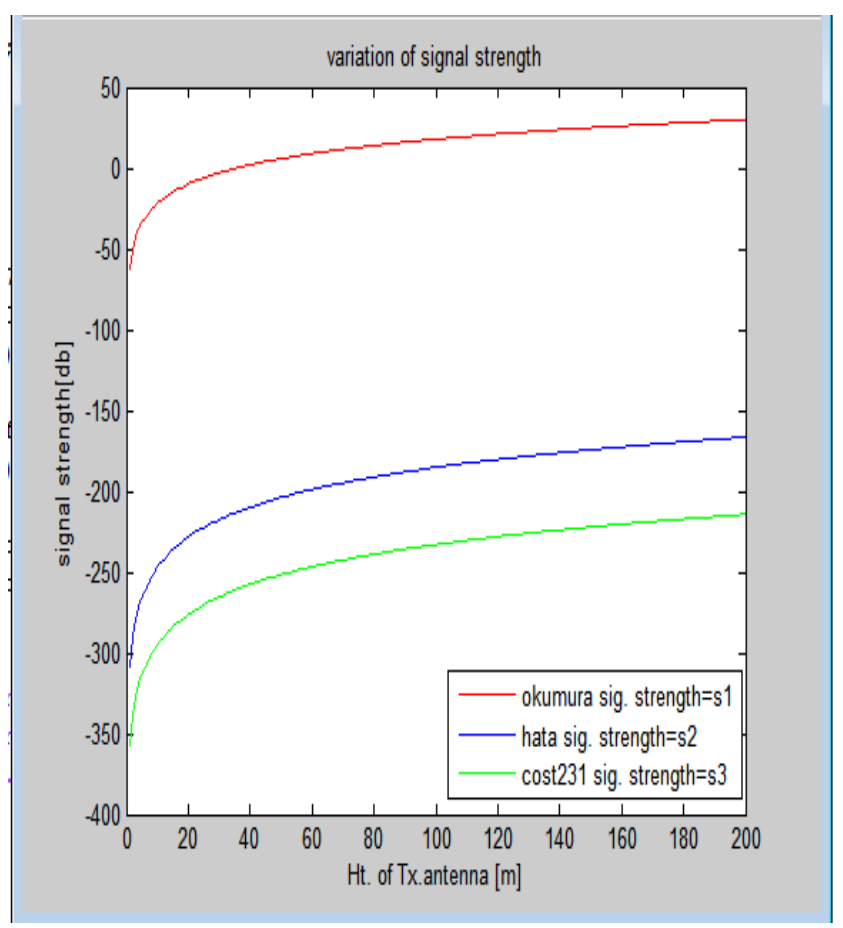

Fig 6. Comparison of signal strength of communication models based on height of transmitter antenna.

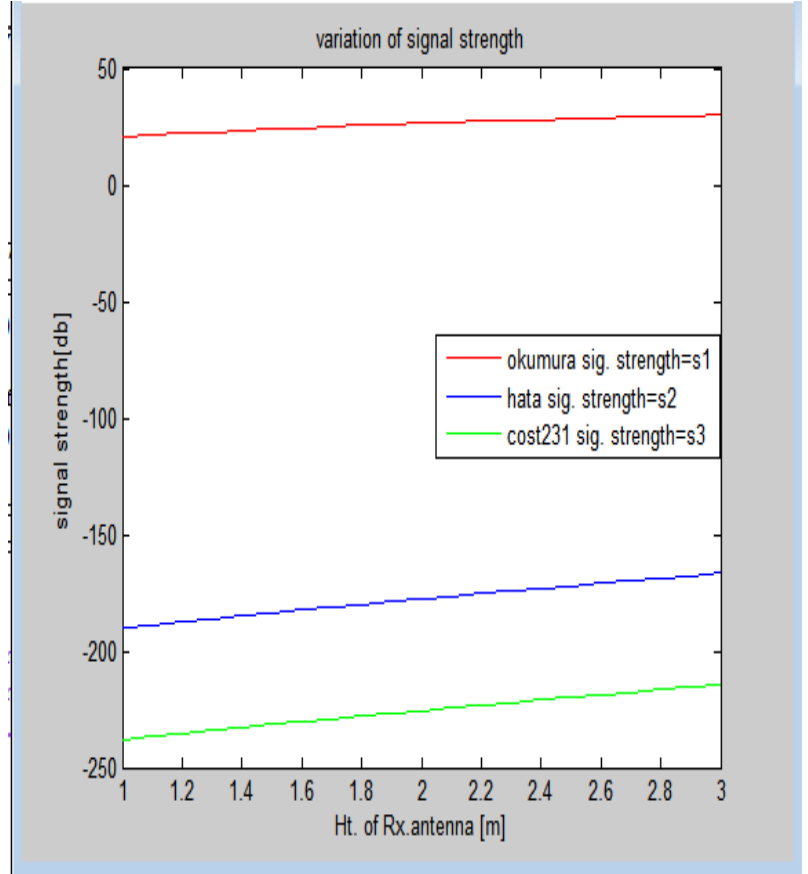

Fig 7. Comparison of signal strength of communication models based on height of receiver antenna

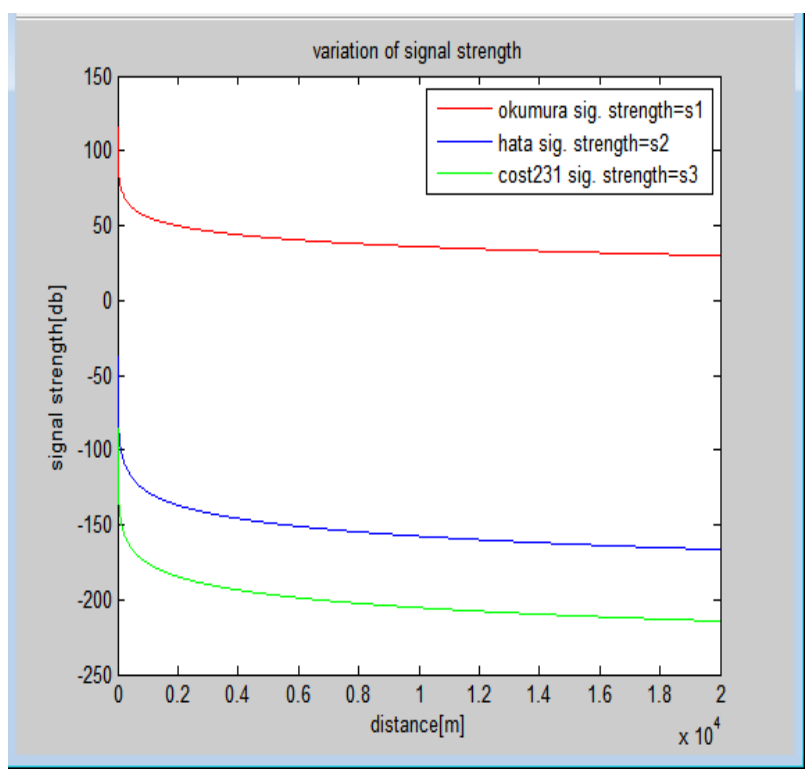

Fig 8. Comparison of signal strength of communication models based on transmission distance

Table 2. Comparison of signal strength of communication models based on coverage distance

\begin{tabular}{|l|l|l|l|}
\hline Distance(km) & $\begin{array}{l}\text { Okumura } \\
\text { model } \\
\text { signal } \\
\text { strength } \\
(\mathbf{d b})\end{array}$ & $\begin{array}{l}\text { Hata } \\
\text { model } \\
\text { signal } \\
\text { strength } \\
(\mathbf{d b})\end{array}$ & $\begin{array}{l}\text { Cost-231 } \\
\text { model } \\
\text { signal } \\
\text { strength } \\
(\mathbf{d b})\end{array}$ \\
\hline 5 & 41.98 & -148.59 & -196.36 \\
\hline 10 & 35.96 & -157.57 & -205.34 \\
\hline 15 & 32.44 & -162.82 & -210.59 \\
\hline 20 & 29.94 & -166.54 & -214.32 \\
\hline
\end{tabular}




\section{Conclusion}

The path loss of Okumura, Hata and Cost 231 models shows decreasing trend with respect to transmitter antenna height and receiver antenna height and increasing trend with respect to transmission distance. Among the communication models Okumura model shows the least path loss and Cost-231 model shows the largest path loss. The signal strength trends are opposite to that of path loss as signal strength with respect to transmitter antenna height and receiver antenna height shows increasing trend and decreasing trend with respect to transmission distance. The signal strength of Okumura model is largest in all the three cases and Cost-231 model shows the least signal strength. Among the three models Hata model shows intermediate results both in case of path loss and signal strength.

\section{REFERENCES}

[1]. T. K. Sarkar, Z. Ji, K. Kim, A. Medour \& M. S. Palma, "A Survey of Various Propagation Models for Mobile Communication", IEEE Antennas and Propagation Magazine, Vol. 45, No. 3, June 2003.

[2] . N. L. B. M. Nordon, "Interface developing for Hata model using Matlab", Universiti Teknologi Malaysia, May 2008.

[3] . Z. Nadir \& M. Idrees Ahmad, "Path loss Determination Using Okumura-Hata Model and Cubic Regression for Missing Data for Oman", Proceeding of IMECS, Vol. 2, 2010.

[4] . H. K. Sharma, S. H. Sahu \& S. Sharma, "Enhanced cost 231 propagations model in wireless network" International journal of computer application (0975 8887) Vol. 19, No. 06, April 2011.

[5] . S. sarooshyari \& N. Madaya, "An Introduction to mobile radio propagation and characterization of frequency bands" wireless comm. Technologies, IEEE, 16:332:559, 1996.

[6] . P. K. Sharma \& R. K. Singh, "Comparative Analysis of Propagation Path loss Models with Field Measured Data", International Journal of Engineering Science and Technology Vol. 2(6), 2010, pp 2008-2013.

[7] . V. S. Abhayawardhana, I. J. Wassell, D. Crosby, M. P. Sellars \& M. G. Brown," Comparison of Empirical Propagation Path Loss Models for Fixed Wireless Access Systems", IEEE, December 2003.

[8] . M. Kumar, V. Kumar \& S. Malik, "Performance and analysis of propagation models for predicting RSS for efficient handoff', International journal of advanced scientific research \& technology, Vol.1, Issue 2, February 2012

[9] . A. Neskovic, N. Neskovic \& G. Paunovic, "Modern approaches in modeling of mobile radio systems propagation environment", IEEE Communications Surveys. 2000.
[10]. P. Schneider, F. Lambrecht \& A. Baier, "Enhancement of the Okumura-Hata propagation using detailed morphological and building data", IEEE, 1996.

[11] . A. H. Ali \& M. R. A. Razak, "Investigation of outdoor fading model over indoor environment", IEEE International Conference on Business, Engineering and Industrial Applications (ICBEIA), 978-1-4577-12807/11/\$26.00 2011.

[12] . J. O. Mark, B. B. Samir \& M. Naufal , "BER Performance evaluation for multicarrier CDMA over generalized $\mathrm{K}-\mu$ fading channels", IEEE symposium on computer and informatics, 2011.

[13] . A. katariya, A. yadav, N. Jain \& G. tomar, "BER performance criteria based on standard IEEE802.11a for OFDM in multipath fading environment", International Conference on Computational Intelligence and Communication Systems, 2011

[14] . S. Nasrzadeh \& M. Jalali, "Channel Estimation and Symbol Detection in AWGN Channel for New Structure of CDMA Signals", Eighth International Conference on Information Technology, 2011.

[15]. H. Kaur, B. Jain \& A. Verma, "Comparative performance analysis of M-ary PSK Modulation Schemes using Simulink", International journal of ECE, IJECT, Vol. 2, Issue 3, September 2011.

[16]. Spectrum Planning Team, "Investigation of Modified Hata Propagation Models", IEEE, April 2001.

[17] . M. A. Masud, M. Samsuzzaman \& M. A. Rahman, "Bit Error Rate Performance Analysis on Modulation Techniques of Wideband Code Division Multiple Access", Journal Of Telecommunication, Volume 1, Issue 2, PP. 22-29, March 2010.

[18]. A. A. Tahir \& F. Zhao, "Performance analysis on modulation techniques of W-CDMA in multipath fading channel “, January 2009.

[19]. O. Grigoriadis \& H. Srikanth Kamath, "Ber Calculation Using Matlab Simulation For OFDM Transmission", IMECS, Vol.2, 2008.

[20]. C. Akkash, "Methods for Path loss Prediction", Report 09067, ISSN 1650-2647, Oct. 2009.

[21]. H. Cavdar," A Statistical Approach to Bertoni Walfisch Propagation Model for Mobile Radio Design in Urban area, IEEE, PP. 279-283, 2001.

[22] . M. Dottling, A. Jahn \& W. Wiesbeck, "A comparison and verification of $2 \mathrm{D}$ and $3 \mathrm{D}$ ray tracing propagation models for land mobile satellite communications", IEEE, 0-7803-6369, PP. 434-437, 2000. 